\title{
ARTICLE OPEN Charge carrier transition in an ambipolar single-molecule junction: Its mechanical-modulation and reversibility
}

Jian Shao ${ }^{1,2}$, Xiaoyue Zhang ${ }^{1,2,3}$, Yun Chen ${ }^{1,2}$ and Yue Zheng ${ }^{1,2,4}$

Precise control from the bottom-up for realizing tunable functionality is of utmost importance to facilitate the development of molecular electronic devices. Until now, however, manipulating charge carriers over single-molecule scale remains intractable. The origin of the problem is that the nature of charge carriers is often hindered by the complexity of the investigated molecular systems. Here, via ab initio simulations, we show a force-modulated and switched ambipolar single-molecule junction with Au/ cyclopropane-1,2-dithiol/Au structure. The cyclopropane ring in the molecule can be opened and closed reversibly and repeatedly by the mechanical force. This structural transition from its closed state to open state enables the ambipolarity in charge carriersfrom p-type to $\mathrm{n}$-type. Analysis of electronic structure reveals unambiguously the force-dependent correlation between $\mathrm{C}-\mathrm{S}$ bond order and the nature of charge carriers. Based on this, we design a binary interconnected junction exhibiting resistance, rectification and negative differential resistance functionalities under mechanical modulation, i.e., loading/unloading or pull/push. This interesting phenomenon provides both illuminating insight and feasible controllability into charge carriers in molecules, and a very general idea and useful approach for single-molecule junctions in practical single-molecule devices.

npj Computational Materials (2016)2:2 ; doi:10.1038/s41524-016-0003-x

\section{INTRODUCTION}

Molecular electronics exploits bottom-up approaches to build functional and tunable devices with minimized dimensions. ${ }^{1}$ The key to this next-generation technology is the design of architecture and the control of transport in each molecular block. ${ }^{2}$ However, this is by no means facile for the small size and complex interface chemistry. ${ }^{3}$ Especially, tuning the charge carriers of the molecule is not as straightforward as in inorganic counterparts. ${ }^{4}$ In top-down Si-based semiconductors, desired $\mathrm{p}$-/n-type semiconductors can be produced by doping impurities with specific valence numbers. ${ }^{5}$ In molecules, however, the charge carrier of molecular transport is accounted inherently on specific electron rich/deficient moiety. ${ }^{6}$ Previous investigations ${ }^{7}$ found that $p$-type molecules includes 1,4-benzenedithiol and 4,4'-diaminostilbene; n-type includes 1,4-benzenedicyanide and 1,2-di(4-pyridyl)ethylene. ${ }^{8}$ Interestingly, oxide counterpart of thiophene, where sulfur lone pairs are engaged in bonding, is electron-deficient. ${ }^{9}$ These examples (see Fig. S1) indicate a native correlation of charge carriers and bond order of non $\mathrm{C}-\mathrm{C}$ bond: $\mathrm{p}$-type molecules equip single bond only (e.g., $\mathrm{C}-\mathrm{S}, \mathrm{C}-\mathrm{N}$ ) while n-type ones equip bond with higher orders (e.g., $\mathrm{O}=\mathrm{S}, \mathrm{C}=\mathrm{N}, \mathrm{C} \equiv \mathrm{N}$ ). However, the bonds in the above individual cases are formed by different atoms, and the influence of bond order has not been confirmed. Therefore, a particular system needs to be designed to exclude the other influences and verify directly the correlation between bond order and $\mathrm{p} / \mathrm{n}$ characteristics.

To manipulate covalent bond precisely in molecules, variable means can be employed, e.g., photonic $^{10}$, mechanical ${ }^{11}$ and electric $^{12}$ activations. Among them, mechanical force shows unique advantages in single-molecule scale. ${ }^{13-16}$ With the development of techniques like atomic force microscope (AFM), scanning tunnel microscope, sonochemical setups, etc., mechanical force can be applied onto specific covalent bond and steer 'forbidden' chemical reactions. ${ }^{17}$ This emerging field, termed as covalent mechanochemistry, ${ }^{18}$ has attracted much attention recently. On the other hand, cyclopropane and its derivatives are suitable samples for investigating bond order-related properties. For example, when force is applied onto polymers with gemdifluorocyclopropane or gem-dichlorocyclopropane ( $g \mathrm{DCC})$ embedded, ring-opening reaction can be triggered. ${ }^{19}$ Meanwhile electrons from the broken $\mathrm{C}-\mathrm{C}$ bond can change the multiplicity of other bonds, ${ }^{20}$ so changes in electron deficiency may be expected.

Here we utilized mechanical forces to manipulate the bond order and the charge carrier of Au/cylcopropane-1,2-dithiol (CPDT)/Au junction. This molecule keeps cyclopropane backbone and has a general representativeness for a wide range of its derivatives. To observe the dynamics process of ring-opening reaction and look into the nature of molecular transport, Car-Parrinello molecular dynamics (CPMD) ${ }^{21}$ and non-equilibrium Green's function (NEGF) investigations have been employed. The molecule can be manipulated reversibly and repeatedly between CPDT and propane-1,3-dithione (PDT), which works as a mechanical flip-flop. The nature of charge carriers is evaluated by thermopower or Seebeck coefficient $S .{ }^{4,22}$ Through ring-opening reaction, $S$ is changed from positive to negative, which means the

\footnotetext{
${ }^{1}$ State Key Laboratory of Optoelectronic Materials and Technologies, School of Physics, Sun Yat-sen University, Guangzhou 510275, China; ${ }^{2}$ Micro\&Nano Physics and Mechanics Research Laboratory, School of Physics, Sun Yat-sen University, Guangzhou 510275, China; ${ }^{3}$ Sino-French Institute of Nuclear Engineering and Technology, Sun Yat-sen University, Zhuhai 519082, China and ${ }^{4}$ Departments of Mechanical Engineering and Civil and Environmental Engineering, School of Engineering, Northwestern University, Evanston, IL 60208, USA

Correspondence: Yue Zheng (zhengy35@mail.sysu.edu.cn)
}

Received: 1 August 2016 Revised: 23 October 2016 Accepted: 2 November 2016

Published online: 09 December 2016 
charge carrier is turned from p-type to n-type. Moreover, this junction excludes other influences on the nature of the charge carrier. So the polarity of this ambipolar molecule ${ }^{23}$ correlates unambiguously to the $\mathrm{C}-\mathrm{S}$ bond order. Based on this, we design a binary interconnected junction that works as a multi-functional triple-state device with each of the block tunable independently.

\section{RESULTS}

Conformational transition in single-molecule junction (SMJ)

Single-molecule structures can be modulated and switched by force-induced ring-opening/closure reactions as shown in Fig. 1. This reaction can turn bond orders and reverse the nature of charge carrier. First we validate the transformation of Au/CPDT/Au junction under external forces through CPMD simulation. The whole process of the steered molecular dynamics has been illustrated as shown in Fig. 2a-c and Movie S1. Briefly, CPDT (i.e., the closed state, structure M1) is pulled into PDT (i.e., the open state, structure $\mathrm{M} 2$ ) through transition state TS. When the gold atom is pulled away from the left electrode, the distance of $\mathrm{C} 1-\mathrm{C} 3$

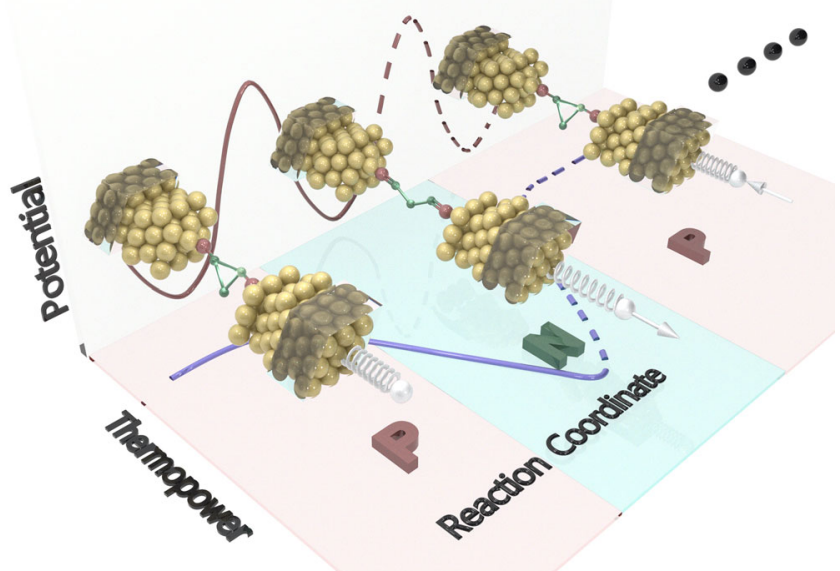

Fig. 1 Schematic illustration of mechanical-modulation and reversibility in a SMJ. Molecular structures of Au/CPDT/Au and Au/PDT/Au junction can be modulated by force-induced ring-opening/-closure reactions. The ring-opening reaction turns $\mathrm{C}-\mathrm{S}$ bond into $\mathrm{C}=\mathrm{S}$ bond and flips the nature of charge carrier from p-type to n-type. The ringclosure reverses the process of ring-opening reaction and flops the nature of charge carriers back to p-type. Yellow for Au; red for S; green for $C$. The hydrogens are removed for simplicity increases from 1.55 to $2.46 \AA$ (see Fig. S3) and the bond breaks (see Fig. S9). During the pulling process, the maximum tensile force on the gold atom (i.e., the 'tip') is $\sim 3.0 \mathrm{nN}$ (see Fig. S4a). Meanwhile the distance of $\mathrm{C}-\mathrm{S}$ bond decreases from 1.77 to 1.64 $\AA$, indicating an increase in bond order. (Literatures ${ }^{24}$ give a single $\mathrm{C}-\mathrm{S}$ bond length of $\sim 1.8 \AA$ and double one of $\sim 1.6 \AA$.) As there is increase in the $\mathrm{C}-\mathrm{S}$ bond order, the electrons from the breaking of $\mathrm{C} 1-\mathrm{C} 3$ bond are paired with electrons from S atoms, so PDT shows no diradical characteristics. Interestingly, PDT can be pushed back into CPDT (see Fig. S3 and Movie S2). When the gold atom in structure M2 is pushed towards the left electrode, the distance of $\mathrm{C} 1-\mathrm{C} 3$ decreases. Finally, $\mathrm{C} 1$ and $\mathrm{C} 3$ rebond and $\mathrm{C}-\mathrm{S}$ bonds are reduced back to single bonds, which makes structure M1. A compressive load of $\sim 1.7 \mathrm{nN}$ on the 'tip' (see Fig. S4b) can facilitate this ring-closure reaction. In other words, this transformation is reversible and mechanical forces can modulate the junction back and forth repeatedly. In addition, both trans- and cis-CPDT show similar reversible structural transformation (see Fig. S2 and Movies S3 and S4). Their peaks in force spectra are also alike (see Fig. S4), indicating similar reaction processes. So only trans-CPDT has been chosen for transport calculations.

To ensure the validity of the results, the free energy surface (FES) of reaction for trans-CPDT is calculated and plotted in Fig. 2d. Two collective variables-CV1 (distance of C1-C3) and CV2 (distance of C1-S1)-are chosen. The FES shows two local minima in (CV1, CV2) coordinates: (1.60, $1.74 \AA$ ) and (2.40, $1.64 \AA$ ), corresponding to the structures $\mathrm{M} 1$ and $\mathrm{M} 2$ in Figs. 2a, respectively. The activation barrier is $\sim 90 \mathrm{~kJ} / \mathrm{mol}$ with no load applied, much lower than the one of $\mathrm{Au}-\mathrm{S}$ dissociation reaction $(\sim 160 \mathrm{~kJ} / \mathrm{mol}){ }^{25}$ It is large enough to forbid thermal activation and small enough to allow $\mathrm{Au}-\mathrm{S}$ bond to survive the mechanical manipulation. The conformational hysteresis under load in Fig. 2e indicates a flip-flop triggered by mechanical force. When the load is applied, the energy barrier is reduced gradually and the reaction happens. The mechanical strength needed to induce the reaction can be calculated by Arrhenius kinetics model. ${ }^{19,26}$ At $2.0 \mathrm{nN}$, the $\mathrm{C} 1-\mathrm{C} 3$ bond breaks; at $-1.5 \mathrm{nN}, \mathrm{C} 1$ and C3 rebond. The forces from the FES give the direct loads on carbon atoms and should be distinguished from the forces given by steered molecular dynamics (3.0 and $-1.7 \mathrm{nN}$ ), which are the loads on the tip. A double-well system like this can work as a bi-stable flip-flop.

The results above reveal the influence of thiol groups and gold electrodes on the ring-opening reaction of cyclopropane. Isolated cyclopropane shows diradical characteristics when the ring is open, which stabilizes under mechanical load. ${ }^{19}$ When cooperated with thiols and gold electrodes, however, the ring- a

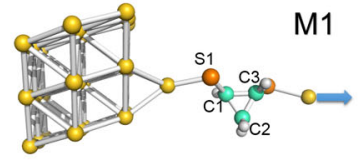

b

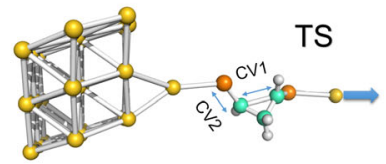

c

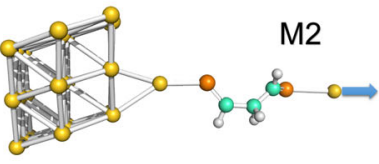

d

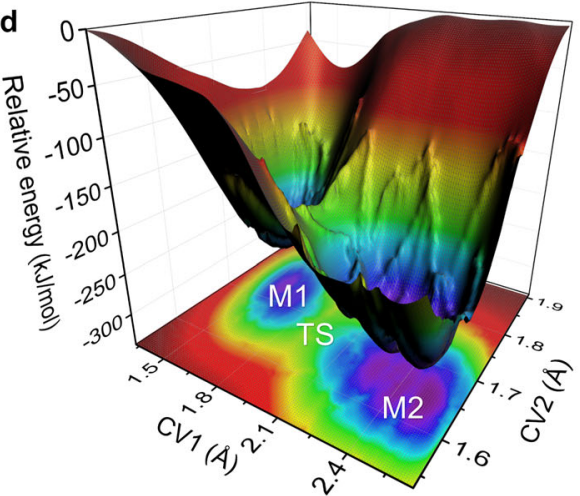

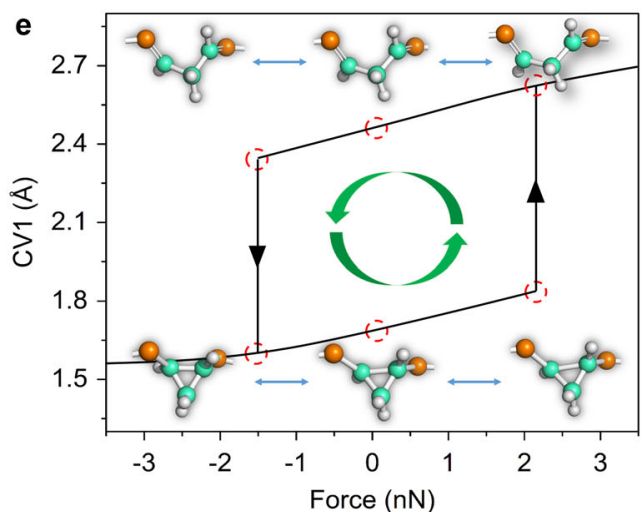

Fig. 2 Force-induced ring-opening and ring-closure reactions. The snapshots of Au/CPDT/Au junction ring-opening process are: local minimum M1 a; transition state TS b; local minimum M2 c. Au, yellow; S, orange; C, green; $\mathrm{H}$, gray (rendered in PyMOL). d The FES for CV1-CV2. $\mathrm{CV} 1$ is the collective variable for the distance of $\mathrm{C} 1-\mathrm{C} 3 ; \mathrm{CV} 2$ is the collective variable for the distance of C1-S1. e The structural hysteresis under mechanical load extracted from force transformed FES (see Fig. S5). The arrows show the directions of conformational changes 

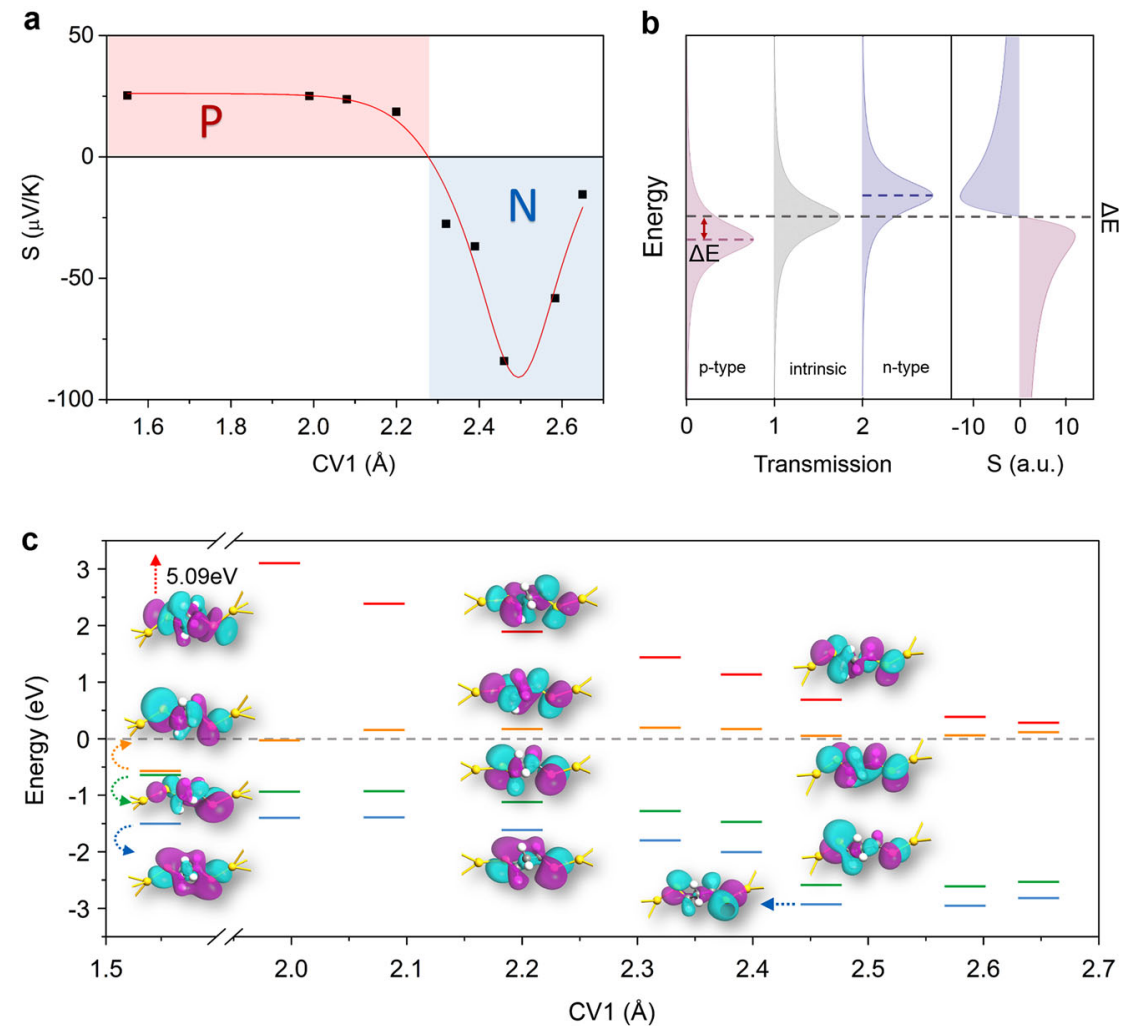

Fig. 3 Charge carriers transition and movement of energy levels in ring-opening reaction. a The thermopower changes during the CV1 (distance of C1-C3) extends from 1.6 to $2.6 \AA$. The junction is p-type for positive thermopower and n-type for negative one. $\mathbf{b}$ Schematic illustration of transmission functions for only one energy level located beneath, on, and above Fermi level which correspond to p-type, neutral, and $n$-type respectively; on the right, the thermopower induced by an individual level for different $\Delta E=-\left(E_{F}-\varepsilon_{i}\right)$ from Eq. (3). c The molecular energy level alignment during CV1 extends from 2.0 to $2.6 \AA$. During the process, the isosurfaces of the orbitals are slightly extended. The gray dashed lines show the Fermi level

opening reaction results in the formation of $\mathrm{C}=\mathrm{S}$ double bonds. As a result, PDT shows no diradical characteristics and can stabilize without mechanical force. Diradical may be observed near TS point during the reaction and discussions are elaborated in supporting information (see Table S1). The absence of diradical in PDT resembles the ring-opening reaction of $g D C C$ where there is no radical in its main product. ${ }^{27}$

The forces required for ring-opening/-closure reactions are in agreement with previous investigations. For trans- $g D C C$, theoretical calculations ${ }^{20}$ predict a C-C breaking force of $2.5 \mathrm{nN}$ and AFM experiments ${ }^{17}$ give a tip force of $2.3 \mathrm{nN}$. Generally, scanning probe microscopy (SPM) experiments ${ }^{28,29}$ give smaller loads of bond rupture compared to those from theoretical calculations. ${ }^{25} \mathrm{We}$ believe it can be attributed to following reasons. First, the time and the route of the bond breaking reaction are both short. The theoretical predictions give the transient maximum force. Nevertheless, the SPM receives the time-averaged data and the maximum is 'smoothed' actually. Thus experimental and theoretical data are not equivalent to this extent. Second, the pulling process may lead to the movement of the surface atoms in the tip and the substrate. The acceleration of the atoms can further modify the load on the tip.

\section{Ambipolarity induced by structural transition}

To investigate the transport nature of the junction, nine snapshots during the ring-opening reaction are picked. Their structures and transmission eigenstates are shown along the CV1 route in Fig. S7. Significant changes can be observed in the morphology of these eigenstates, indicating different molecular orbitals are involved in transport. These snapshots are typical samplings of the ringopening process. Their thermopowers are extracted from transmission spectra (see Fig. S8) and plotted in Fig. 3a. CV1 is taken as the measurement of conformational change of these snapshots. When CV1 increase from 1.6 to $2.1 \AA$, the thermopower $S$ keeps steady near $25 \mu \mathrm{V} / \mathrm{K}$. Then it drops quickly from positive to negative values when CV1 increases from 2.2 to $2.3 \AA$. This flip in sign of $S$ indicates the charge carriers of the junction are turned from p-type to n-type. In n-type region, the giant thermoelectric effect and its ratio can be observed and summarized. ${ }^{30}$ Here the giant thermoelectric ratio (GTR) can be defined as the ratio of maximum value to minimum value for the thermopower $S$. At 2.5 $\AA$, the thermopower reaches as large as $-100 \mu \mathrm{V} / \mathrm{K}$. When the distance increases further, the thermopower decreases rapidly to less than $-20 \mu \mathrm{V} / \mathrm{K}$. The GTR is larger than 5, enabling the mechanical force as an effective tool for enhancing the low Seebeck coefficient in unloaded molecular electronics. ${ }^{31}$ The signs of thermopower associated with the two stable conformations M1 and M2 (local minima in FES) are opposite. Therefore, the junction can be flip-flopped between p-type and n-type states by mechanical load, which means the junction exhibits ambipolarity.

The change of thermopower can be explained by the movement of molecular orbital level alignment during the reaction. To begin with, we will analyze the contribution of each molecular orbital to thermopower. At single-particle limit, the thermopower can be expressed $a^{22}$

$$
S=\left.\frac{\pi^{2} k_{B}^{2} T}{3 e} \frac{d \ln [\mathcal{T}(E)]}{d E}\right|_{E_{F}}
$$




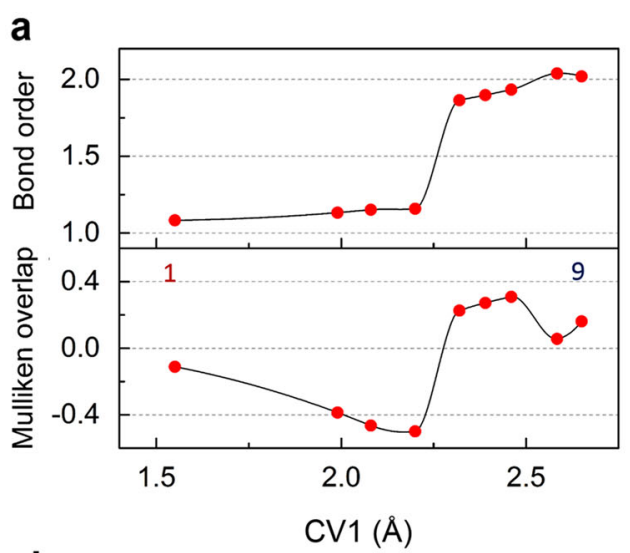

b

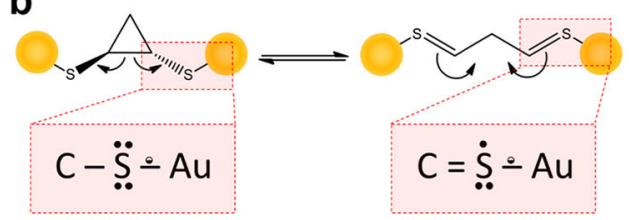

C

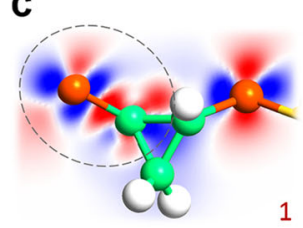

1
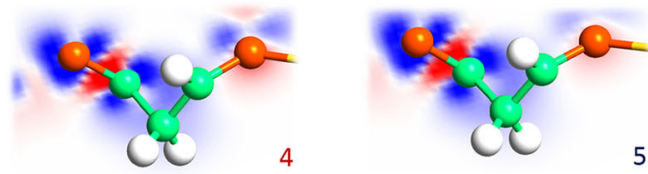

2
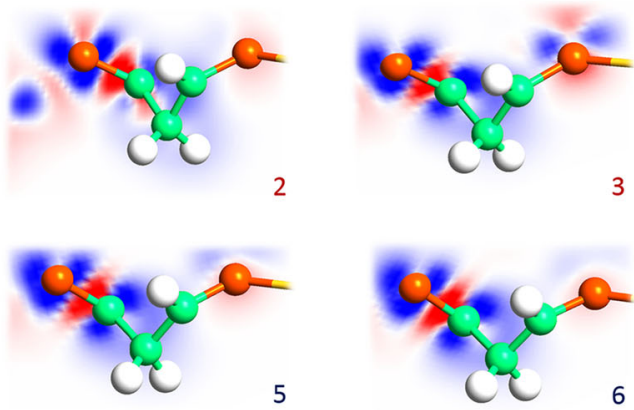

6
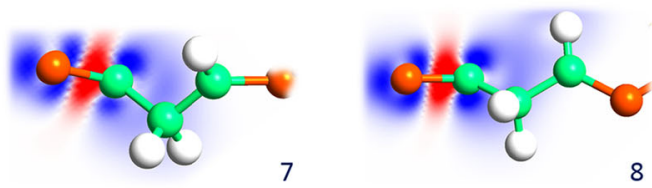

8

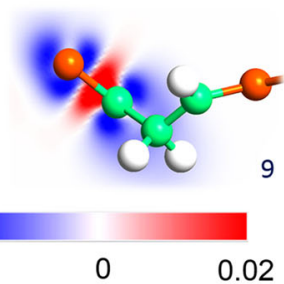

Fig. 4 C-S bond order transition and electron distribution in ring-opening reaction. a The change of bond order and Mulliken overlap of $\mathrm{C} 1-\mathrm{S} 1$ as CV1 (the distance of $\mathrm{C} 1-\mathrm{C} 3$ ) increases from 1.6 to $2.6 \AA$. b Electron redistribution near $\mathrm{S}$ atom in ring-opening/-closure reaction. The solid dot is the local electron from S; the semi-hollow dot is the delocalized partial electrons from Au; the line is the bond between atoms. c The change of difference charge density in the C1-S1 bond region for 9 snapshots

where $T$ is the mean temperature of the two contacts, $E$ is the energy, $E_{\mathrm{F}}$ is the Fermi level, and $\mathcal{T}(E)$ is the energy-resolved transmission. Each transmission peak is induced by molecular orbital levels and can be fitted approximately with Lorentzian function,

$\mathcal{T}(E)=\sum_{i=1}^{n} \frac{\Gamma_{1} \Gamma_{2}}{\left(E-\varepsilon_{i}\right)^{2}+\frac{\left(\Gamma_{1}+\Gamma_{2}\right)^{2}}{4}}$,

where $\varepsilon_{i}$ is the energy of the molecular level, and $\Gamma_{1}$ and $\Gamma_{2}$ are the broadenings by left and right contacts, respectively. Substituting Eq. (2) into Eq. (1), one arrives at expression of thermopower contributed from all orbitals:

$S=-\frac{2 \pi^{2} k_{\mathrm{B}}^{2} \Gamma_{1} \Gamma_{2} T}{3 e \mathcal{T}\left(E_{\mathrm{F}}\right)} \sum_{i=1}^{n} \frac{E_{\mathrm{F}}-\varepsilon_{i}}{\left[\left(E_{\mathrm{F}}-\varepsilon_{i}\right)^{2}+\frac{\left(\Gamma_{1}+\Gamma_{2}\right)^{2}}{4}\right]^{2}}$

As illustrated in Fig. $3 b$, the level above the Fermi level gives negative contributions and the one beneath the Fermi level gives positive contributions. Within this approximation, $S$ is related non-monotonically to $\Delta E=-\left(E_{\mathrm{F}}-\varepsilon_{i}\right)$ and has extrema at finite $\Delta E$.

Then we analyze movement of molecular orbital level alignment during the reaction illustrated in Fig. 3c. Before the reaction, the transport of $\mathrm{Au} / \mathrm{CPDT} / \mathrm{Au}$ junction is dominated by yellow (HOMO) and green (HOMO-1) levels. As C1-C3 distance increases gradually, the red level of CPDT moves towards the Fermi level, while the green and blue levels move away from the Fermi level. The yellow level moves up across the Fermi level at first, and then moves downwards slightly. This switch, from HOMO to LUMO, can be explained by the delocalization of electrons on $\pi$-orbitals of $\mathrm{C}-\mathrm{S}$ bonds. More specifically, as the $\mathrm{C}-\mathrm{C}$ bond is stretched gradually, the electrons are redistributed to the $\pi$-orbitals of $\mathrm{C}-\mathrm{S}$ bonds (see Fig. 4b). For the conjugate nature and the coupling with the electrodes, these electrons are delocalized throughout the junction and the projection on the molecule is little. Finally, when CPDT transforms to PDT, transport of the junction is dominated by yellow (LUMO) and red (LUMO + 1) levels. According to Eq. (3), Au/CPDT/Au junction has positive $S$ and is thus p-type. On the contrary, Au/PDT/Au junction has negative $S$ and is thus n-type. Moreover, this typical model can also explain the giant thermoelectric effect in Fig. 3a. During the reaction, the red level moves down towards Fermi level. Its contribution to $S$ will increase first and then decrease accounting on the $S \sim \Delta E$ relationship.

\section{Correlation of bond order and charge carrier}

It is observed that the order of C-S bond has a strong correlation to the sign of charge carriers. As illustrated in Fig. $4 \mathrm{a}$, the bond order of $\mathrm{C} 1-\mathrm{S} 1$ evolves from single to double during the ringopening reaction. The Wiberg bond order endures a slow increase from 1.08 to 1.16 when $\mathrm{C} 1-\mathrm{C} 3$ length increases from 1.5 to $2.2 \AA$. Then it surges up to 1.86 at $2.3 \AA$ and oscillates in a small amplitude for longer lengths. Meanwhile, the Mulliken overlap of $\mathrm{C} 1-\mathrm{S} 1$ turns positive values from negative ones at the same point. These changes are in excellent agreement with the reverse of the charge carrier. To understand the correlation, we follow the basic ideas of Si-based semiconductors and propose a simple model concerning bond orders. Consider an alkane backbone. Each carbon atom bonds to four other atoms, which forms a tetrahedron analogous to silicon. For thiol and thione anchoring groups, the electron distribution can be simplified by electron dot diagrams in Fig. 4b. In this scenario, atom is considered 'neutral' in electronic transport when each of the four directions is covalently bonded or forms lone pairs. When the thiol group anchors to Au electrode, Au donates about extra 0.5 electrons to it. Thus the thiol group is electron rich and acts as an electron donor, making the junction p-type. On the other hand, the extra electrons from Au are not enough to 'neutralize' thione group. So thione group is electron deficient or an electron acceptor and the junction is n-type.

During the reaction, the electron distribution is more complicated and we refer to the difference charge density around the $\mathrm{C} 1-\mathrm{S} 1$ bond illustrated in Fig. 4c. When $\mathrm{C} 1-\mathrm{C} 3$ bond breaks (from snapshot 2 to 4 ), the electrons redistribute to the space between $C-S$. However, the bond order does not increase significantly, indicating these electrons are not 'well-bonded'. So the moiety is electron rich and the junction is p-type. At snapshot 5, the difference charge density does not change much but the 
a

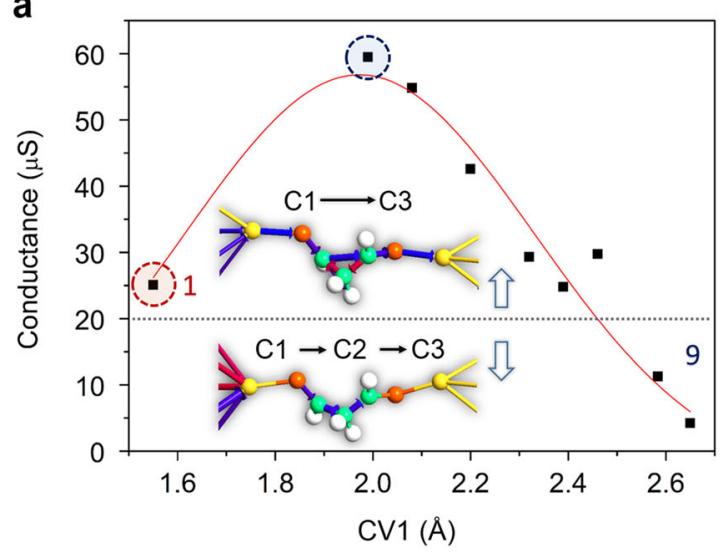

b

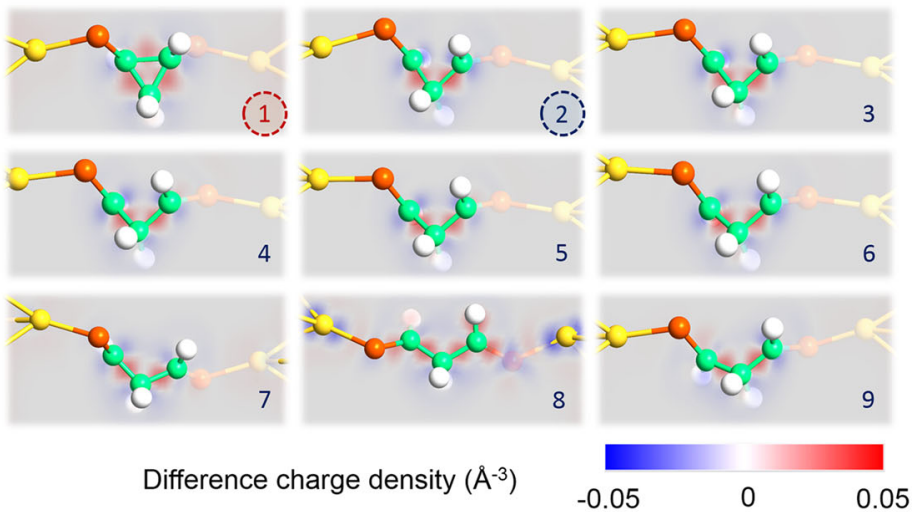

Fig. 5 Relationship between the bond break and the conductance change of SMJ. a The conductance change during the distance of $\mathrm{C} 1-\mathrm{C} 3$ extends from 1.6 to $2.6 \AA$. The conductance increases from 1.6 to $2.0 \AA$ decreases from $2.0-2.6 \AA$. The inset shows the changes in transmission pathway (contributions from the left eletrode). For points above the blue dashed line, the pathway is $\mathrm{C} 1 \rightarrow \mathrm{C} 3$; for points beneath the line, the pathway is $\mathrm{C} 1 \rightarrow \mathrm{C} 2 \rightarrow \mathrm{C} 3$. b The difference charge density change from snapshot 1 to snapshot $\mathbf{9}$

\section{a}

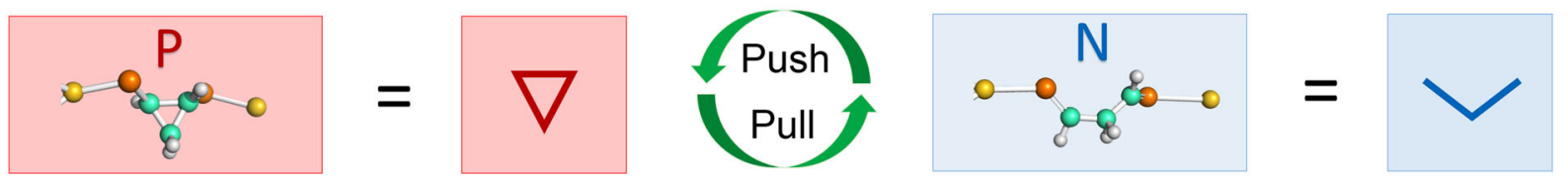

b

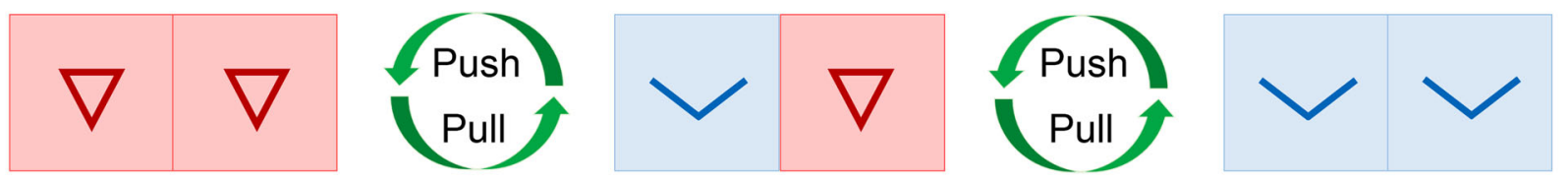

C

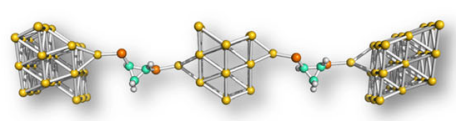

f

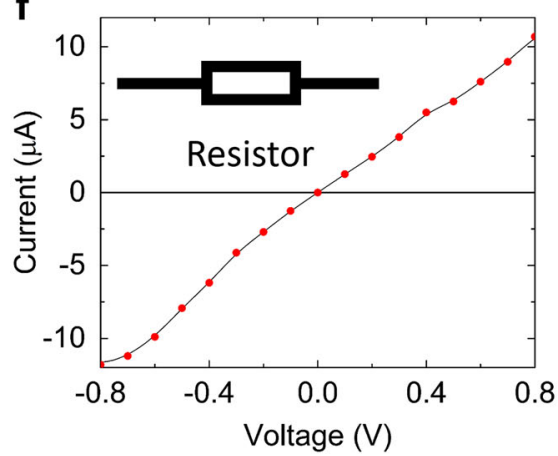

d

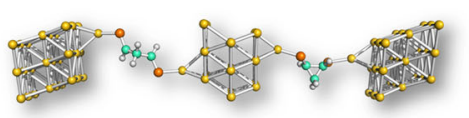

g

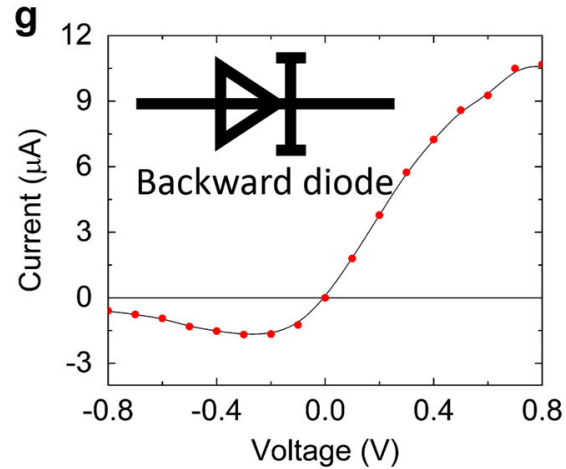

e

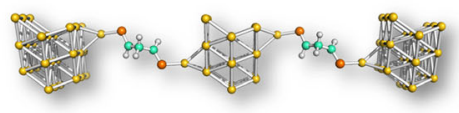

h

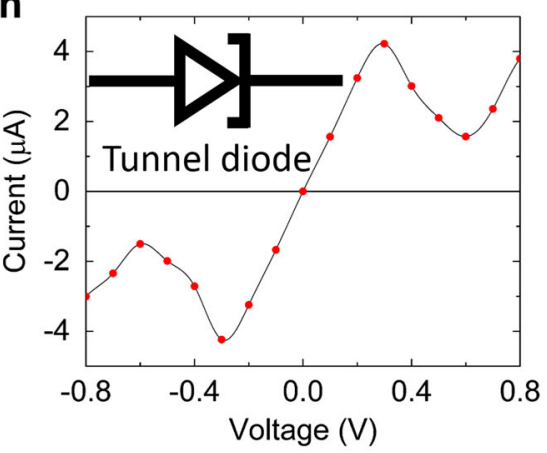

Fig. 6 Structural change and kinds of I-V curves of SMJ during the mechanical manipulation. Schematic illustration of the structural switch of unary $\mathbf{a}$ and binary $\mathbf{b}$ junction during the mechanical manipulation. $\mathbf{c}-\mathbf{h}$ Structures and corresponding I-V curves of the three states of binaryconnected junction. Each works as a resistor, a backward diode and a tunnel diode respectively

bond order surges, which means the extra electrons form double bond with $\mathrm{S}$ orbitals. Then the moiety becomes electron deficient and the junction becomes n-type. This model can also be applied to explain the charge carriers of other Au-anchored molecules terminated with, e.g., thiol, amino, pyridyl and cyano groups (see Fig. S10). For other electrodes, the difference in the relative position of electrode Fermi level and frontier molecular orbital may influence the results. ${ }^{32}$ Our previous investigation also suggests that HOMO level of alkanedithiol will shift to different extents when thiol group anchors to $\mathrm{Au}$ and $\mathrm{Ag}$ electrodes. ${ }^{33}$ However, it is beyond the scope of this work.

Force modulating conductance of SMJ

The conductance of the junction can also be modulated by mechanical force. As shown in Fig. 5, the conductance increases as 
a

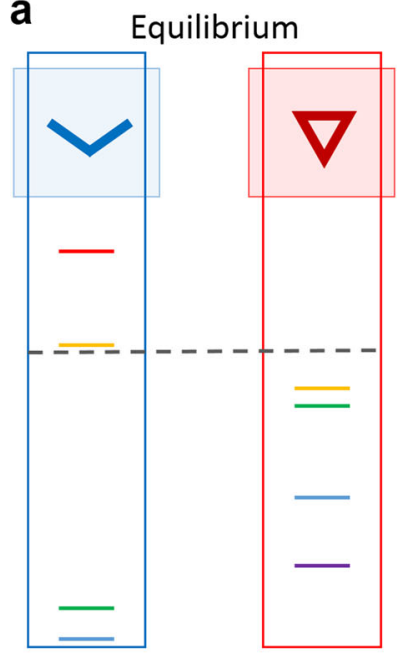

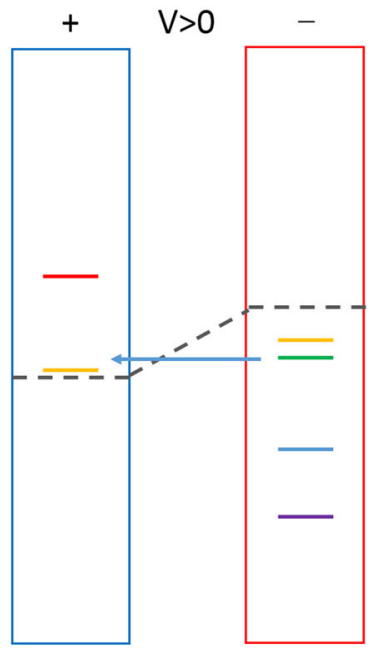

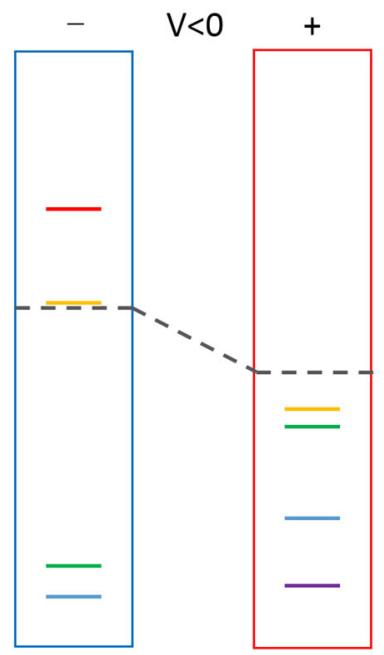

b
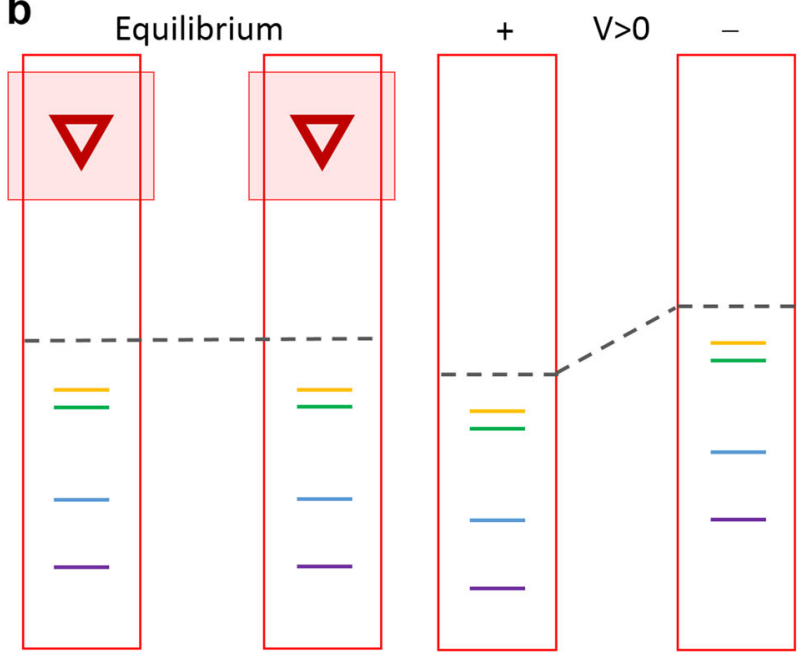

C
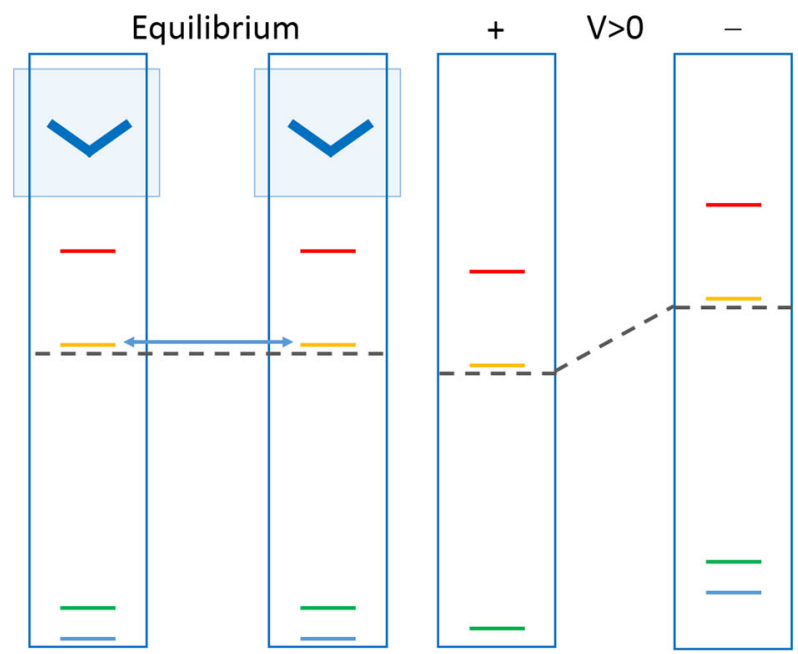

Fig. 7 Molecular energy level alignment for backward diode, resistor and tunnel diode effects of SMJ. Molecular energy level alignments at different voltages are shown for PDT\&CPDT a, CPDT\&CPDT $\mathbf{b}$ and PDT\&PDT c junctions respectively. The arrows show the directions of electronic resonant tunneling. The color of the levels corresponds to those in Fig. $3 \mathrm{c}$

CV1 increases from 1.5 to $2.0 \AA$ and decreases from 2.0 to $2.6 \AA$. The increase of conductance in $1.5-2.0 \AA$ is associated with the stretching of bent bond in CPDT. Literally, cyclopropane has shorter but weaker $\mathrm{C}-\mathrm{C}$ bond than regular alkane bonds. When cooperated with thiol, the bond length of $\mathrm{C} 1-\mathrm{C} 3$ is even shorter. In M1 conformation (i.e., CV1 = $1.5 \AA$ ), the maximum electron density between two carbon atoms does not correspond to the internuclear axis. When CV1 is $2.0 \AA$, the bent bond is stretched into 'line' and the orbitals are no longer 'distorted'. In this conformation, the overlap of two orbitals is comparable to M1 conformation. On the other hand, a portion of electrons from $\mathrm{C} 1-\mathrm{C} 3$ bond redistribute to the $\pi$-orbital of $\mathrm{C}-\mathrm{S}$ bonds. These electrons are delocalized, which pushes the molecular level and the induced transmission peak towards Fermi level (see Fig. 3 and Fig. S8). Thus the transmission with CV1 of $2.0 \AA$ is larger than that with CV1 of $1.5 \AA$. After $2.0 \AA$, the delocalization of partial electrons could not compensate the decrease of orbital overlap in C1-C3 bond, so the transmission reduces. At $\sim 2.5 \AA$, the $\mathrm{C} 1-\mathrm{C} 3$ bond breaks totally and the pathway changes from $\mathrm{C} 1 \rightarrow \mathrm{C} 3$ to $\mathrm{C} 1 \rightarrow \mathrm{C} 2 \rightarrow \mathrm{C} 3$. It is obvious that the tunneling conductance will decrease because the pathway extends much longer. To conclude, the change of conductance is the result of two competing factors: the orbital overlap in C1-C3 bond and the delocalization of the redistributed electrons during the pulling process.
A binary multi-functional molecular junction

The force-modulated ambipolarity of the SMJ enables design of multi-functional devices by interconnections. Here we show in Fig. 6, a simple example of a binary-connected molecular junction manipulated by mechanical force. A symmetric Au/CPDT\&CPDT/ $\mathrm{Au}$ junction (see Fig. $6 \mathrm{c}$ ), where the two p-type molecules are connected by three layers of Au with one adatom on each side, is a resistor (see Fig. 6f).

When the CPDT\&CPDT structure is pulled, one of the propane ring may open firstly. Thus the original $p-p$ structure has now evolved into a p-n structure. As illustrated in Figs. $6 \mathrm{~d}$ and $\mathrm{g}$, the asymmetric Au/CPDT\&PDT/Au junction works as a typical donoracceptor rectifier. ${ }^{34}$ The rectification ratio is as large as $\sim 20$ at $0.8 \mathrm{~V}$. To confirm the rectification, a different connecting structure has also been investigated and similar results have been observed (see Fig. S11). One may have noticed that the junction has the opposite rectification direction compared with standard p-n junctions, which makes it a backward diode. ${ }^{35}$ To explain this phenomenon, we plot the level diagrams of the molecular junction in Fig. 7a. According to the level alignment, the transport is dominated by the LUMO and LUMO + 1 of PDT and HOMO and HOMO-1 of CPDT. Under positive voltage, the bias pushes these molecular levels closer. When their energies are close enough, 
PDT's HOMO and HOMO-1 donate electrons to CPDT's LUMO and LUMO + 1 through tunneling, resulting in a high conductance. In this scenario, the p-type PDT becomes an electron donor and n-type CPDT becomes an electron acceptor. This phenomenon is analogous to Zener breakdown in inorganic semiconductors. Compared with inorganic counterparts, molecular junction has much higher tunneling current for its shorter barriers. Under negative voltage, on the contrary, the bias pulls farther these molecular levels of PDT and CPDT. So the tunneling probability decreases. Accordingly, the PDT\&CPDT junction shows rectification as a backward diode.

When the junction is pulled further on the other side, the other CPDT will endure ring-opening reaction and it becomes an $n-n$ structure (see Fig. 6e). This junction shows negative differential resistance (NDR) (see Fig. 6h). Unlike $p$-p junction where Fermi level are far from the HOMO and LUMO levels, n-n junction have both LUMO levels located near Fermi level (see Figs. 7b,c). At zero voltage, resonant tunneling is strong. When applied voltages are large, the LUMO levels will shift in opposite directions and resonant transport is reduced for the large energy gap. Similar NDR effect in single-molecule break junctions has been reported ${ }^{36}$ where the two moieties of the molecule are connected by a non-conjugated organic bridge. To conclude, in CPDT\&CPDT, PDT\&CPDT and PDT\&PDT structures, multi-phenomena should be modulated directly or switched repeatedly due to the change of bond order induced by mechanical loads.

\section{DISCUSSION}

In conclusion, our study shows that ring-opening reaction can bring unique tunable features to the emerging molecular electronics. By mechanically opening and closing the ring, the type of charge carriers can be switched reversibly. To provide a clear and general insight into the transport nature, complex structural design for applied purpose is abandoned. This ambipolar SMJ reveals clearly the correlation between $\mathrm{C}-\mathrm{S}$ bond order and the nature of charge carriers under mechanical load. Further work has to be done to exploit this 'dynamic doping' process in constructing functional blocks of molecular electronics. Through proper design, these building blocks can be organized into functional devices. A particularly attractive feature is that each block of the interconnected junction can be controlled by mechanical force independently. Thus a tunable binary junction is proposed, which can be mechanically switched to be a resistor, a $p-n$ junction or NDR. More functions are expected in complicated molecular networks. However, the difference in single-molecule and solid-state devices should be handled carefully. Besides, the reversal of thermopower and large GTR carve more possibilities in applications like energy conversion and temperature sensing.

\section{METHODS}

Structure of the SMJ

All calculations were carried out using Perdew-Burke-Ernzerhof functional. ${ }^{37}$ The structural flip-flop transition was simulated with CPMD in CPMD code. Troullier-Martins pseudopotentials ${ }^{38}$ were used for $\mathrm{C}, \mathrm{H}$ and $S$ and an 11 electron Goedecker scalar-relativistic dual-space pseudopotential for $\mathrm{Au}^{39,40} \Gamma$-point sampling with 100 Ry plane wave energy cutoff was enough for efficient convergence and was in accordance with previous report. The system was attached to Nose-Hoover thermostat to prevent undesirable heat transfer problems. A hexagonal periodic supercell with lattice vectors of $8.65 \times 8.65 \times 30.0 \AA^{3}$ was employed. The left slab was ranged in three layers of $3 \times 3$ gold atoms, with the first layer fixed to bulk positions. The molecule was connected to a gold adatom on each side through a sulfur atom and attached to the electrode. For bi-molecule interconnected structure, two CPDT molecules were connected through gold layers or a gold atom. Then a hexagonal supercell of $8.65 \times 8.65 \times$
$40.0 \AA^{3}$ was selected. The atoms were relaxed until forces are less than $0.02 \mathrm{eV} / \AA ̊$

\section{Process of mechanical load on SMJ}

During the pulling process, ${ }^{41}$ the right adatom was constrained and pulled away in $\mathrm{z}$ axis at a steady speed of $12 \AA /$ ps to more than $4 \AA$ at $300 \mathrm{~K}$. After relaxation, the adatom was pushed back in $z$ axis at the same speed. The potential energy surface was calculated by metadynamics simulations. ${ }^{42,43}$ Two collective variables were selected: $\mathrm{CV} 1$, the distance of $\mathrm{C} 1-\mathrm{C} 3$ and $\mathrm{CV} 2$, the distance of C1-S1. Their masses were set to $20 \mathrm{amu}$, the $k a^{\prime}$ s to 0.2 and $\Delta s$ is 0.1 . $\Delta s^{\|}$fluctuates between 0.02 and 0.04 and $\mathrm{W}$ between 2.5 and $21 \mathrm{~kJ} / \mathrm{mol}$. V $(t)$ was updated every $0.005 \mathrm{ps}$. For efficient metadynamics, constraints were applied to the structure of anchoring groups and the movement of the Au 'tip' atom in parallel to the 'substrate' surface was not allowed (see Fig. S5 for details).

\section{Transport properties of SMJ under mechanical load}

The structural snapshots of pulling and pushing process were extracted for calculations of transport properties. The right electrode was added to form symmetric-electrode junction. The periodic cell was fixed in $a$ and $b$ lattices and allowed to relax in $c$ direction. The atoms in both electrodes were allowed to relax to a tolerance of $0.02 \mathrm{eV} / A ̊$. The relaxation was implemented in Vienna ab-initio Simulation Package ${ }^{44}$ and plane wave basis set with an energy cutoff of $500 \mathrm{eV}$ for all atoms. Г-point sampling was maintained. The bond order information was calculated in natural atomic orbital basis with Gaussian program. LANL2DZ basis set was employed for $\mathrm{Au}$ and $6-31 \mathrm{G}^{*}$ basis set was employed for $\mathrm{C}, \mathrm{H}$ and $\mathrm{S}$. Transport calculations were performed within density functional theory ${ }^{45}$ based NEGF approach using Atomistix ToolKit. ${ }^{46}$ Here a linear combination of atomic orbitals basis set with energy cutoff of 75 Ry was chosen. All atoms in the molecule were modeled with double- $\zeta$ plus polarization basis sets and the metal atoms were modeled with single- $\zeta$ plus polarization basis sets for both accuracy and efficiency. Self-consistency was achieved using a $4 \times 4 \mathrm{k}$-point sampling in the two-dimensional Brillouin zone. Then the energy resolved transmission spectra were calculated every $0.01 \mathrm{eV}$ with an $8 \times 8 \mathrm{k}$-mesh.

\section{ACKNOWLEDGEMENTS}

The authors gratefully acknowledge the financial support of NSFC (Nos. 11402312, 11474363, 11232015) and the National Key Basic Research Program of China (No. 2015CB351905). Y.Z. also thanks support by the Fundamental Research Funds for the Central Universities to Micro\&Nano Physics and Mechanics Research Laboratory, Fok Ying Tung Foundation, Science and Technology Innovation Project of Guangdong Provincial Education Department and Guangdong Natural Science Funds for Distinguished Young Scholar.

\section{AUTHOR CONTRIBUTIONS}

$\mathrm{YZ}$ initiated and performed this work and manuscript. JS, $\mathrm{XYZ}$ and $\mathrm{YZ}$ conceived and designed the basic idea and structures. J.S. performed the simulations. JS, XYZ, YC and $Y Z$ analyzed the results of simulations. JS, XYZ and $Y Z$ co-wrote the manuscript. All authors contributed to discussion and reviewed the manuscript.

\section{COMPETING INTERESTS}

The authors declare that they have no competing interests.

\section{REFERENCES}

1 Sun, L. et al. Single-molecule electronics: from chemical design to functional devices. Chem. Soc. Rev. 43, 7378-7411 (2014).

2 Joachim, C. \& Ratner, M. A. Molecular electronics: some views on transport junctions and beyond. Proc. Nat. Acad. Sci. USA 102, 8801-8808 (2005).

3 Flood, A. H., Stoddart, J. F., Steuerman, D. W. \& Heath, J. R. Chemistry. Whence molecular electronics? Science 306, 2055-2056 (2004).

4 Reddy, P., Jang, S. Y., Segalman, R. A. \& Majumdar, A. Thermoelectricity in molecular junctions. Science 315, 1568-1571 (2007).

5 Shockley, W. The theory of p-n junctions in semiconductors and p-n junction transistors. Bell Syst. Tech. J. 28, 435-489 (1949).

6 Anthony, J. E. et al. n-Type organic semiconductors in organic electronics. Adv. Mater. 22, 3876-3892 (2010). 
7 Aradhya, S. V. \& Venkataraman, L. Single-molecule junctions beyond electronic transport. Nat. Nanotechnol. 8, 399-410 (2013).

8 Widawsky, J. R., Darancet, P., Neaton, J. B. \& Venkataraman, L. Simultaneous determination of conductance and thermopower of single molecule junctions. Nano Lett. 12, 354-358 (2012).

9 Dell, E. J. et al. Molecular length dictates the nature of charge carriers in singlemolecule junctions of oxidized oligothiophenes. Nat. Chem. 7, 209-214 (2015).

$10 \mathrm{Jia}, \mathrm{C}$. et al. Covalently bonded single-molecule junctions with stable and reversible photoswitched conductivity. Science 352, 1443-1445 (2016).

11 Hickenboth, C. R. et al. Biasing reaction pathways with mechanical force. Nature 446, 423-427 (2007).

12 Schwarz, F. et al. Field-induced conductance switching by charge-state alternation in organometallic single-molecule junctions. Nat. Nanotechnol. 11, 170-176 (2016).

13 Marszalek, P. E. et al. Mechanical unfolding intermediates in titin modules. Nature 402, 100-103 (1999).

14 Franco, I. et al. Mechanically activated molecular switch through single-molecule pulling. J. Am. Chem. Soc. 133, 2242-2249 (2011).

15 Paulsson, M., Krag, C., Frederiksen, T. \& Brandbyge, M. Conductance of alkanedithiol single-molecule junctions: a molecular dynamics study. Nano Lett. 9, 117-121 (2009).

16 Zhang, X. Y. et al. Structure-dependent electrical conductivity of protein: its differences between alpha-domain and beta-domain structures. Nanotechnology 26, 125702 (2015)

17 Wang, J. et al. Inducing and quantifying forbidden reactivity with single-molecule polymer mechanochemistry. Nat. Chem. 7, 323-327 (2015)

18 Ribas-Arino, J. \& Marx, D. Covalent mechanochemistry: theoretical concepts and computational tools with applications to molecular nanomechanics. Chem. Rev. 112, 5412-5487 (2012)

19 Lenhardt, J. M. et al. Trapping a diradical transition state by mechanochemical polymer extension. Science 329, 1057-1060 (2010).

20 Dopieralski, P., Ribas-Arino, J. \& Marx, D. Force-transformed free-energy surfaces and trajectory-shooting simulations reveal the mechano-stereochemistry of cyclopropane ring-opening reactions. Angew. Chem. Int. Ed. 50, 7105-7108 (2011).

21 Car, R. \& Parrinello, M. Unified approach for molecular dynamics and densityfunctional theory. Phys. Rev. Lett. 55, 2471-2474 (1985).

22 Paulsson, M. \& Datta, S. Thermoelectric effect in molecular electronics. Phys. Rev. $B$ 67, 241403(R) (2003).

23 Diez-Perez, l. et al. Ambipolar transport in an electrochemically gated singlemolecule field-effect transistor. Acs Nano 6, 7044-7052 (2012).

24 Allen, F. H. et al. Tables of bond lengths determined by X-ray and neutron diffraction. Part 1. Bond lengths in organic compounds. J. Chem. Soc., Perkin Trans 2, S1-S19 (1987)

25 Gronbeck, H., Curioni, A. \& Andreoni, W. Thiols and disulfides on the $\mathrm{Au}(111)$ surface: The headgroup-gold interaction. J. Am. Chem. Soc. 122, 3839-3842 (2000).

26 Beyer, M. K. The mechanical strength of a covalent bond calculated by density functional theory. J. Chem. Phys. 112, 7307-7312 (2000).

27 Wollenhaupt, M., Krupicka, M. \& Marx, D. Should the Woodward-Hoffmann rules be applied to mechanochemical reactions? Chemphyschem. 16, 1593-1597 (2015).
28 Xue, Y., Li, X., Li, H. \& Zhang, W. Quantifying thiol-gold interactions towards the efficient strength control. Nat. Commun. 5, 4348 (2014).

29 Grandbois, M. et al. How strong is a covalent bond? Science 283, 1727-1730 (1999).

30 Ohta, H. et al. Giant thermoelectric seebeck coefficient of a two-dimensional electron gas in SrTiO3. Nat. Mater. 6, 129-134 (2007).

31 Chang, W. B. et al. Controlling the thermoelectric properties of thiophene-derived single-molecule junctions. Chem. Mater. 26, 7229-7235 (2014).

32 Hasegawa, T. \& Takeya, J. Organic field-effect transistors using single crystals. Sci. Technol. Adv. Mater. 10, 024314 (2009).

33 Shao, J. et al. Length-dependent rectification and negative differential resistance in heterometallic n-alkanedithiol junctions. Rsc. Adv. 5, 13917-13922 (2015).

34 Aviram, A. \& Ratner, M. A. Molecular rectifiers. Chem. Phys. Lett. 29, 277-283 (1974).

35 Amos, S. W. \& Amos, R. Newnes Dictionary of Electronics (Elsevier Science, 2002).

36 Perrin, M. L. et al. Large negative differential conductance in single-molecule break junctions. Nat. Nanotechnol. 9, 830-834 (2014).

37 Perdew, J. P., Burke, K. \& Ernzerhof, M. Generalized gradient approximation made simple. Phys. Rev. Lett. 77, 3865-3868 (1996).

38 Troullier, N. \& Martins, J. L. Efficient pseudopotentials for plane-wave calculations. Phys. Rev. B 43, 1993-2006 (1991).

39 Hartwigsen, C., Goedecker, S. \& Hutter, J. Relativistic separable dual-space Gaussian pseudopotentials from $\mathrm{H}$ to Rn. Phys. Rev. B 58, 3641-3662 (1998).

40 Krüger, D. et al. Pulling monatomic gold wires with single molecules: an Ab initio simulation. Phys. Rev. Lett. 89, 186402 (2002).

41 Curioni, A. et al. Density functional theory-based molecular dynamics simulation of acid-catalyzed chemical reactions in liquid trioxane. J. Am. Chem. Soc. 119, 7218-7229 (1997)

42 lannuzzi, M., Laio, A. \& Parrinello, M. Efficient exploration of reactive Potential energy surfaces using car-parrinello molecular dynamics. Phys. Rev. Lett. 90, 238302 (2003).

43 Laio, A. \& Parrinello, M. Escaping free-energy minima. Proc. Nat. Acad. Sci. USA. 99, 12562-12566 (2002).

44 Kresse, G. \& Hafner, J. Ab initio molecular dynamics for liquid metals. Phys. Rev. $B$ 47, 558-561 (1993).

45 Kohn, W. \& Sham, L. J. Self-consistent equations including exchange and correlation effects. Phys. Rev. 140, A1133-A1138 (1965).

46 Brandbyge, M. et al. Density-functional method for nonequilibrium electron transport. Phys. Rev. B 65, 165401 (2002).

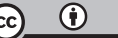

This work is licensed under a Creative Commons Attribution 4.0 International License. The images or other third party material in this article are included in the article's Creative Commons license, unless indicated otherwise in the credit line; if the material is not included under the Creative Commons license, users will need to obtain permission from the license holder to reproduce the material. To view a copy of this license, visit http://creativecommons.org/licenses/by/ $4.0 /$

(c) The Author(s) 2016

Supplementary Information accompanies the paper on the npj Computational Materials website (doi:10.1038/s41524-016-0003-x). 